\title{
Cuidados primários em saúde na atenção à população em situação de rua
}

\author{
Primary health care in attention to the population in street situation
}

\author{
Chen Laura ${ }^{1}$ \\ Orcid : https://orcid.org/0000-0002-8210-5724
}

Marcelo Medeiros Salles ${ }^{3}$

Orcid: https://orcid.org/0000-0002-8092-8081

Lilian Machado Torres ${ }^{5}$

Orcid: https://orcid.org/0000-0002-7510-7078

\author{
Alline Dias Cruz ${ }^{2}$ \\ Orcid: https://orcid.org/0000-0002-1685-9624 \\ Rosângela Durso Perillo ${ }^{4}$ \\ Orcid: https://orcid.org/0000-0003-4791-2317 \\ Shirley Pereira Almeida ${ }^{6}$ \\ Orcid: https://orcid.org/0000-0003-3236-3095
}

\begin{abstract}
Resumo
Introdução: o fenômeno situação de rua constitui-se um desafio para sua compreensão, dimensionamento, diagnósticos diversos e para a definição de estratégias para enfrentar as condições de vulnerabilidade, entre elas as de saúde. Objetivo: descrever as ações desenvolvidas pelos profissionais de saúde e as dificuldades vivenciadas no atendimento à população em situação de rua. Métodos: pesquisa qualitativa, a partir de entrevistas com profissionais de saúde de uma Unidade Básica de Saúde de Belo Horizonte-MG, referência para atendimento a essa população. O conteúdo dos depoimentos foi analisado pelo referencial de Bardin. Resultados: os profissionais identificam ações realizadas, a partir do acolhimento sistematizado com vistas à criação de vínculos e abordagem dos principais problemas de saúde. Realizam ações preventivas necessárias e encaminham ou acionam outros serviços, como albergues ou centros pop. Sentem-se satisfeitos e gratificados, apesar de perceberem a necessidade de maior articulação com outros pontos da rede assistencial diante de atendimentos mais complexos ou específicos. Também enfrentam dificuldades para a redução de danos em várias dimensões como vacinação, saúde bucal e geral, saúde mental e prevenção e tratamento de doenças crônicas e infectocontagiosas. Emergiram duas categorias: Identificando ações desenvolvidas pelos profissionais da Atenção Primária e Enfrentando dificuldades e desafios no atendimento a essa população. Conclusões: é preciso ultrapassar preconceitos e conhecer a situação de rua para sua implicação nos cuidados prestados na Atenção Primária, apesar dos dificultadores. Capacitação dos profissionais e planejamento multi e interdisciplinar, em rede, concorrem para qualidade na captação dos indivíduos e sua inserção efetiva nos serviços de saúde.
\end{abstract}

Palavras-chave: atenção primária à saúde; pessoas em situação de rua; pessoal de saúde

\section{Abstract}

Introduction: individuals living on the street are a worldwide phenomenon and a real challenge in terms of their understanding, their dimensioning, diverse and necessary

\footnotetext{
${ }^{1}$ Faculdade Ciências Médicas de Minas Gerais, Brasil. E-mail: laurachen04@gmail.com

${ }^{2}$ Faculdade Ciências Médicas de Minas Gerais, Brasil. E-mail: allinediaz@hotmail.com

${ }^{3}$ Faculdade Ciências Médicas de Minas Gerais, Brasil. E-mail: salles-marcelo@uol.com.br

${ }^{4}$ Secretaria Municipal de Saúde de Belo Horizonte/MG, Brasil. E-mail: rosangeladurso.perillo@gmail.com

${ }^{5}$ Instituto de Previdência dos Servidores do Estado de Minas Gerais, Brasil. E-mail: lilian.torres0806@gmail.com

${ }^{6}$ Faculdade Ciências Médicas de Minas Gerais, Brasil. -mail: shirley.almeida@cienciasmedicasmg.edu.br
} 
assessments as well as for the definition of strategies for coping with the conditions of vulnerability, including health. Objective: to describe the actions developed by health professionals and the difficulties experienced when attending the homeless population. Méthods: qualitative research, based on interviews with health professionals at a Basic Health Unit in Belo Horizonte-MG, a reference for attending this population. The content of the testimonies was analyzed using the Lawrence Bardin method. Results: professionals identify their actions based on the systematic reception that promotes the creation of bonds and the approach to the main health problems. They carry out necessary preventive actions and refer or activate other services, such as hostels or pop centers. They feel satisfied and gratified, despite the certainty that greater articulation with the care network is necessary in the face of more complex or specific health care. They also face difficulties in harm reduction in various dimensions such as vaccination, oral and general health, mental health and prevention and treatment of chronic and infectious diseases. Two categories emerged: Identifying actions developed by Primary Care professionals and Facing difficulties and challenges in serving this population. Conclusions: it is necessary to overcome prejudices and know the true dimension of being on the street for its involvement in the care provided in Primary Care, despite the difficulties. Professional training and multi and interdisciplinary planning, in a network, contribute to quality in attracting individuals and their effective insertion in health care services.

Keywords: primary health care; homeless persons; health personal

\section{Introdução}

O fenômeno população em situação de rua (PSR) refere-se a um grupo negligenciado em relação às políticas públicas e às estratégias para seu enfrentamento, fato que se constitui desafio para diversos países ${ }^{1-2}$.

As dificuldades para a compreensão desse fenômeno iniciam-se na própria definição, aceita mundialmente e, ao mesmo tempo, adaptada aos cenários regionais. $\mathrm{O}$ alinhamento conceitual é fundamental para os censos da PSR, análises e comparações de dados que possibilitem a proposição de políticas públicas $^{1-2}$. Em 2015, o Institute of Global Homelessness define PSR como a 'população sem acesso a uma habitação minimamente adequada', conceituação mais ampla que abrange um universo maior de pessoas ${ }^{3}$.

A Organização das Nações Unidas estima 100 milhões de pessoas em situação de rua (SR), no mundo ${ }^{4}$. Em sua maioria, a PSR é composta de homens em torno dos 50 anos, com baixa escolaridade, solteiros e divorciados, com problemas de saúde que envolvem ferimentos autoprovocados, lesão na cabeça por perda de consciência, epilepsia, tuberculose (TB), transtornos mentais, abuso de álcool, tabaco e outras $\operatorname{drogas}^{5-7}$.

No Brasil, detecta-se o aumento significativo da PSR composta por indivíduos excluídos da sociedade, sem direitos sociais, em situação de indigência ou pobreza e em degradado estado de saúde $^{8}$. A contagem oficial em nível nacional não é realizada, o que reforça a invisibilidade desta população e dificulta o planejamento e a instituição de políticas públicas direcionadas às suas reais necessidades. Apesar disso, os dados podem ser conhecidos a partir de estimativas obtidas por compilação, análise e estatística de números oficiais coletados por mais de 5.500 municípios. Um estudo da estimativa da PSR, realizado em 2020 pelo Instituto de Pesquisa Econômica Aplicada revelou um crescimento de $140 \%$ desde setembro de 2012 a março de 2020, algo em torno de 222 mil pessoas, sendo que a maior parte $(81,5 \%)$ está em municípios com mais de 100 mil habitantes, principalmente nas regiões Sudeste $(56,2 \%)$, Nordeste $(17,2 \%)$ e Sul $(15,1 \%)^{9}$.

Tal quadro compõe um fenômeno complexo, multifacetado, inserido na lógica capitalista e relacionado às desigualdades sociais de distribuição de 
renda e bens sociais e à política econômica excludente e ineficiente. Como resultado, revela-se um histórico de internações em casas de recuperação e detenção, orfanatos e hospitais psiquiátricos, por sofrimento mental, TB, epilepsia e uso de drogas. Diante disso, 44\% dos indivíduos em SR procuram hospitais e serviços de urgência para suas demandas e $27 \%$ buscam as Unidades Básicas de Saúde (UBS) ${ }^{8,10-12}$.

Pesquisa realizada em Minas Gerais pelo Polo de Cidadania da Universidade Federal de Minas Gerais, em 2020, a partir de dados do Cadastro Único para Programas Sociais do Governo Federal, identificou 18.011 pessoas em SR, sendo que 12 municípios concentram 70\% (12.804) dessa população, em um total de 853 municípios $^{13}$. Em Belo Horizonte foram identificadas 9.114 pessoas na mesma situação, ou seja $50,6 \%$ do total do estado, o que revela um aumento significativo ao se comparar com os dados do terceiro censo realizado em 2013, no qual existiam 1.827 pessoas nesta situação. A maior parte de homens $(88 \%)$, com idades entre 31 e 50 anos $(67 \%)$ e pardos ou negros (80\%), o que revela a desigualdade racial presente ${ }^{13-14}$. Dentre os principais motivos que levaram esta população à condição de rua estão 0 desemprego $(29,8 \%)$, os conflitos familiares $(29,1 \%)$ e o alcoolismo/drogas $(35,5 \%)^{15}$.

As condições de vulnerabilidade, para além das psicossociais produtoras de sofrimentos físicos e emocionais, porém, indissociáveis entre si, representam um desafio para a efetivação de políticas de saúde $^{4,11}$. Tais condições demonstram que o adoecimento é um processo dinâmico e reflexo da interação entre os aspectos biológicos, sociais, culturais, econômicos, políticos e os determinantes sociais de saúde (DSS) ${ }^{4}$.

Estratégias adotadas por alguns municípios brasileiros para o cuidado com a saúde da PSR foram a implementação de algumas políticas, serviços e ações como, por exemplo, a educação popular de rua e projetos de redução de danos (RD), ao final de 1980, na cidade de Santos. Acrescenta-se o "Programa A Gente na Rua", em São Paulo e Salvador, em $1990^{11,14}$.

Em outros municípios, foram instituídas equipes de saúde da família (SF), a partir da implantação da Estratégia de SF (ESF), como modelo de cuidado preconizado para o nível da Atenção Primária à Saúde (APS), porta de entrada da Rede de Atenção à Saúde (RAS). Esse foi o caso do município de Belo Horizonte que implantou, em 2002, a primeira equipe de SF para PSR (eSF-PSR), seguido de São Paulo, Rio de Janeiro, Porto Alegre, Curitiba e Recife ${ }^{11,14-15}$.

Em 2009, instituiu-se a Política Nacional para a PSR (PNPSR), iniciativa nacional de reconhecimento dos direitos do então definido grupo de indivíduos em vulnerabilidade econômica, familiar, social, sem delimitação de espaço de sobrevivência e que, por sua vez, utiliza de lugares públicos ou de acolhimento para sua permanência provisória e/ou permanente $^{8,11,15}$.

A PNPSR, além da universalidade, igualdade e equidade, propõe como princípios o respeito à dignidade da pessoa humana, o direito à convivência familiar e comunitária, a valorização e respeito à vida e à cidadania, o atendimento humanizado $\mathrm{e}$ o respeito às condições sociais e diferenças de origem, raça, idade, nacionalidade, gênero, orientação sexual e religiosa, com atenção especial às pessoas com deficiência. Ressalta-se, também, as diversas diretrizes desta política, que além de determinar a promoção dos direitos civis, políticos, econômicos, sociais, culturais e ambientais desta população, sob responsabilidade do Estado para sua elaboração e financiamento, impõe que cada nível de governo articule e integre as políticas públicas em seu âmbito de gestão. Outras diretrizes importantes envolvem incentivar e apoiar a organização da referida população e sua participação nas diversas instâncias de formulação, controle 
social, monitoramento e avaliação do que for implementado, respeitar às especificidades de cada território e aproveitar as potencialidades e recursos locais e regionais no planejamento, execução e monitoramento das respectivas políticas, além de implantar e ampliar ações educativas para superar preconceitos e capacitar os trabalhadores para a qualificar o atendimento a esta população ${ }^{8}$.

Os princípios e diretrizes previstos na PNPSR tem como propósito garantir os direitos dos indivíduos em SR tais como o acesso amplo, simplificado e seguro aos serviços e aos programas de saúde, educação, previdência, assistência social, moradia, segurança, cultura, esporte, lazer, trabalho e renda. Para tanto, deve garantir a formação e capacitação permanente de profissionais e gestores para desenvolver políticas públicas intersetoriais, transversais e intergovernamentais direcionadas a esta situação, produzir dados e indicadores que reflitam a realidade e que subsidiem propostas concretas e objetivas voltadas às necessidades detectadas. Ressalte-se, ainda, a importância de articular o Sistema Único de Assistência Social (SUAS) e o Sistema Único de Saúde (SUS) para qualificar a oferta de serviços, sua estruturação de acolhimento temporário e a implementação de centros de referência especializados a este público ${ }^{8}$.

A instituição de serviços e equipamentos sociais como os de acolhimento institucional temporário e os Centros Pop também se configuram como conquistas na SR, implementados a partir de políticas públicas. Albergues, abrigos e casas de passagem, integrantes da proteção social especial de alta complexidade do SUAS visam oferecer atendimento integral e garantir condições de estadia, convívio e endereço de referência para acolher a PSR, migrantes e/ou pessoas oriundas de áreas de risco geológico ${ }^{11,15}$.

Os centros de referência especializados para PSR, também denominados 'Centros Pop', referidos anteriormente, são unidades socioassistenciais municipais integrantes da proteção social especial de média complexidade do SUAS, devem funcionar em dias úteis, mas podem estender seus horários em feriados, finais de semana e período noturno. Os 'Centros Pop' visam atender algumas das necessidades prementes de pessoas em tal situação, ou seja, a preservação da integridade e a autonomia, a redução das violações dos direitos socioassistenciais, seus agravamentos ou reincidência e a $\mathrm{RD}$ provocados por situações violadoras de direitos. A partir da escuta qualificada busca a compreensão do contexto social, cultural e histórico de cada pessoa, além de elaborar o plano de acompanhamento individual e/ou familiar. Tais centros, como espaço de referência para o convívio grupal, social e o desenvolvimento de relações de afetividade, respeito e solidariedade pretendem contribuir para a construção de novos projetos de vida, respeitando as escolhas dos usuários, possibilitar condições de acolhida na rede socioassistencial e promover ações para a reinserção familiar e/ou comunitária. Outrossim, questões básicas estão igualmente asseguradas, como alimentação, espaço para higiene pessoal e a guarda de pertences, ao se considerar a identidade e subjetividade das pessoas para o fortalecimento do seu protagonismo e participação social $^{11,15}$.

Além da PNPSR, destacam-se a criação do Comitê Intersetorial de Acompanhamento e Monitoramento da PNPSR, espaço de diálogo entre representantes desta população e os de diversos setores do Estado, bem como a instituição do Comitê Técnico de Saúde da PSR e as equipes dos Consultórios na Rua (eCR) para melhorar a capacidade de resposta às necessidades existentes. Igualmente, definiram-se as equipes de apoio multidisciplinares, vinculadas à Coordenação de Saúde Mental (SM) e à atenção básica, criadas para realizar atividades como busca ativa e cuidado, de 
forma itinerante, aos usuários de álcool, crack e outras drogas. Todas as ações ou atividades devem ser realizadas de forma compartilhada e integrada às UBS e, quando necessário, junto aos Centros de Atenção Psicossocial, serviços de urgência e emergência e outros pontos da RAS ${ }^{11,15-}$ 18 .

Observa-se que, de alguma forma, a PSR é acolhida pelo SUS, mesmo diante de supostos dificultadores para $o$ atendimento e acompanhamento pelos profissionais da saúde ou assistência social e para o desenvolvimento das práticas cotidianas direcionadas a tais indivíduos.

Torna-se fundamental fazer emergir, pelo olhar do profissional de saúde, as ações direcionadas à PSR ao se considerar suas características singulares, vulnerabilidade social e da própria saúde, além de identificar as possíveis dificuldades vividas em tal abordagem.

Os resultados possibilitarão discussões e proposições de estratégias de saúde e cuidados direcionados a esse grupo e poderão contribuir para a qualificação das equipes com vistas à maior articulação da RAS, a partir de estratégias voltadas a esse fenômeno. Enfim, poderão otimizar recursos disponíveis, bem como identificar outras necessidades.

Diante deste cenário o estudo teve por objetivo descrever as ações desenvolvidas pelos profissionais de saúde e as dificuldades vivenciadas no atendimento à PSR, na perspectiva dos trabalhadores.

\section{Materiais e Métodos}

Abaixo segue a sugestão das subseções e níveis que devem estar contidos na seção Material e Métodos.

\section{Amostra e tipo de estudo}

Pesquisa qualitativa sobre as ações desenvolvidas por profissionais de saúde na atenção à PSR em uma UBS. Tal abordagem considera a relação dinâmica entre o mundo real e o indivíduo, com ênfase na subjetividade, que não se traduz em números ${ }^{19-20}$.
O cenário foi uma UBS de referência para atendimento à PSR, situada na regional Centro-Sul do município de Belo Horizonte, MG. Participaram os profissionais das eSF-PSR e Saúde Bucal (SB) para PSR (eSB-PSR), eCR e das equipes de apoio à $\mathrm{SF}$ para este grupo como psiquiatra, assistente social, psicólogo e enfermeiro.

\section{Delineamento da pesquisa}

A coleta dos depoimentos ocorreu entre maio e junho de 2015, após a aprovação nos Comitês de Ética em Pesquisa da Faculdade Ciências Médicas de Minas Gerais e da Prefeitura de Belo Horizonte, sob os números $954.096 \mathrm{e}$ 1.041.652, respectivamente.

Utilizou-se um roteiro semiestruturado de entrevista que permitiu a livre expressão de ideias e que incluiu, em sua primeira parte, dados referentes ao perfil dos participantes. As questões norteadoras propostas contemplaram informações sobre as ações de saúde e intersetoriais desenvolvidas junto à PSR, as dificuldades enfrentadas no cotidiano e que outros profissionais estariam envolvidos na atenção ao público específico, além da discriminação do papel desempenhado em tais ações.

\section{Critérios de Inclusão e Exclusão}

Foram incluídos profissionais de saúde da referida unidade de saúde com, pelo menos, um ano de atuação no atendimento à PSR. Foram excluídos aqueles em férias ou licença, ou ausentes por quaisquer motivos, no período da coleta de informações. A seleção dos participantes foi intencional, pois em pesquisa qualitativa permite-se selecionar pessoas que podem contribuir para $o$ estudo, considerados informantes-chave e ricos de informações ${ }^{20}$. A amostra totalizou 16 participantes identificados por letras e números, de P1 a P16.

\section{Procedimentos}


Após a aproximação do pesquisador junto à UBS e aos participantes procedeu-se à assinatura do Termo de Consentimento Livre e Esclarecido, em reuniões agendadas pelo gestor na unidade, para as devidas explicações sobre o projeto e seus objetivos. Nestas oportunidades definiu-se o local e o horário para a realização das entrevistas gravadas, no próprio serviço de saúde. Nos encontros estavam presentes três alunas do último ano do Curso de Enfermagem que coletaram os depoimentos sob orientação e treinamento de três professores, dois mestres e um doutor, respectivamente. A cada encontro, com duração média de 20 minutos, estavam na sala destinada à coleta de dados apenas uma aluna e o entrevistado. Em seguida, os depoimentos foram transcritos na íntegra, paralelamente à coleta, e analisados pelo grupo de pesquisadores, o que permitiu constatar a saturação dos dados na entrevista do $16^{\circ}$ participante, não sendo necessária a inclusão de novos profissionais $^{20}$.

Ao final procedeu-se à leitura e releitura do material no sentido de se buscar a convergência do que foi exposto. Foram identificados os temas emergentes para a análise de conteúdo, realizada à luz do referencial de Bardin $^{21}$, método que permite o estudo do que está contido nas mensagens expressas. Para tal, procedeuse a avaliação inicial para a organização das etapas seguintes, mediante o primeiro contato com os depoimentos. Em seguida, o passo de escolha e recorte das unidades de registro, denominada de fase de exploração do material colhido. A terceira etapa incluiu a inferência e interpretação dos resultados obtidos ${ }^{21}$. As etapas descritas aconteceram com a presença de todos os pesquisadores.

\section{Resultados}

O cenário do estudo possui 23 profissionais de saúde envolvidos diretamente no atendimento à PSR. Pelo critério de saturação de dados participaram
16 trabalhadores, sendo $75 \%$ do sexo feminino e $69 \%$ com formação de nível superior, com destaque para enfermeiros e psicólogos. Do total, $50 \%$ deles têm, em média, de um a cinco anos de trabalho junto aos indivíduos em SR.

Da eSF-PSR participaram dois técnicos de enfermagem, dois agentes comunitários de saúde (ACS) e um médico generalista. Esta equipe, no momento da realização da pesquisa, estava defasada em relação ao profissional enfermeiro que se aposentou e a dois ACS que solicitaram exoneração do cargo. A eSB-PSR é composta por um cirurgião-dentista, um técnico de SB, um auxiliar de SB. Apenas o auxiliar não participou da pesquisa, pois estava de licença médica. Os outros profissionais participantes deste estudo compõem a equipe de apoio à eSF-PSR, a saber, dois enfermeiros, dois psicólogos, um assistente social, um médico psiquiatra. Da eCR participaram o assistente social, o enfermeiro e o psicólogo. $\mathrm{O}$ agente redutor de danos e o arte educador não compareceram nos dias agendados para a entrevista.

A partir do processo de análise dos depoimentos coletados emergiram duas categorias: Identificando ações desenvolvidas pelos profissionais da APS e Enfrentando dificuldades e desafios no atendimento a essa população.

\section{Identificando ações desenvolvidas pelos profissionais da APS junto à PSR}

Algumas ações destacadas pelos entrevistados são de âmbito geral, outras, mais específicas, de acordo com a categoria profissional ou a equipe.

Os profissionais destacaram a identificação e o cadastramento do usuário, o acompanhamento e o atendimento da PSR durante as visitas no território-rua como o momento em que é possível realizar ações preventivas, verificar as necessidades e identificar as situações para encaminhamentos ou acionamento de outros serviços e equipamentos: 
"Acompanhamos o morador de rua, cadastramos, encaminhamos para albergues, casas de apoio e, quando nos deparamos com um doente, acionamos a ambulância e tentamos encaminhar para a UPA (referindo-se à Unidade de Pronto Atendimento)." [P14]

"... ações de prevenção de doenças. Acompanhamento, diagnóstico, levantamento de dados, identificação para acompanhamento da equipe. Necessidade de atendimento clínico, odontológico, psiquiátrico ou psicológico." [P5]

"Contamos com apoio $d a$ abordagem de rua, do Consultório na Rua, da Polícia Militar." [P15].

Diversas ações realizadas na UBS e em outros serviços foram citadas como o albergue e os centros pop. Os profissionais percebem o acolhimento como principal estratégia para a construção de vínculos. Elencaram a investigação do estado vacinal passado e presente e dos sintomas respiratórios para a detecção precoce de $\mathrm{TB}$, além de testes para diagnóstico de HIV e hepatite:

"Visitas ... ao albergue, ao centro pop, busca ativa e abordagens na rua." [P15]

"Acolhimento, educação em saúde, testes rápidos para HIV, sifilis e hepatite $B$. Aconselhamento pré e pós-testes. A tuberculose... acontece muito em situação de rua, porque são vulneráveis, desnutridos e vivem em aglomerados. Quando diagnosticados, muitos abandonam o tratamento. Eles são acolhidos na unidade, avaliamos a demanda e os encaminhamos. As questões sociais $e$ de saúde mental são encaminhadas para a assistente social. Analisamos também o estado vacinal." [P13].

"A equipe trabalha muito com tuberculose. Estamos atentos a esse fato." [P6]

Os participantes afirmam que a forma de acolhimento da PSR possibilita o diálogo, a reflexão e o compartilhamento do desejo de sair da rua. Para o profissional é preciso a habilidade para a escuta e a sensibilidade em relação à situação:

"Essa acolhida possibilita que as pessoas reflitam sobre a melhor qualidade de vida. Muitos procuram clínicas de tratamento para se livrar das drogas ou emprego para sair do albergue, voltar à vida normal e não ficar em situação de rua. Porque é muito triste." [P13]

Um dos profissionais da eSF-PSR ressaltou a satisfação pelo cuidado prestado, pela possibilidade de ajudar, ouvir e acompanhar as histórias de vida, além da gratificação mediante os resultados:

"Acompanhamos adolescentes, gestantes e idosos que falam de sua vida, é satisfatório conversar, ouvir. É gratificante trabalhar com a população de rua quando você vê os resultados." [P14]

No entanto, a necessidade da articulação da rede intersetorial, em função da especificidade da PSR e das demandas para além do setor saúde, foi destacada pelos profissionais:

"Trabalhamos a intersetorialidade. Não é uma população qualquer que tem problema de saúde! Tem questões vinculadas ao social, associadas a outros serviços. Não se trabalha separado." [P4]

Os depoentes revelaram que identificar a demanda da PSR e as ações de cuidado necessárias possibilita acionar profissionais de acordo com suas competências, além das intervenções multiprofissionais, diante de atendimentos mais complexos ou específicos, em outros pontos da RAS:

"Em processos mais delicados, que precisam do acompanhamento médico, acionamos o médico ou o enfermeiro." [P14]

"Abordamos os usuários, analisamos a demanda. Caso esteja machucado a enfermeira faz curativos ou encaminhamos para a UPA, Centro de Saúde ou Hospital." [P2]

A eSB-PSR relacionou o adoecimento bucal e a saúde em geral, 
além de enfatizar as ações de cuidado para reduzir danos:

"Fazemos todo tipo de ação inerente à odontologia. Educação em saúde, prevenção, atuação clínica e promoção de saúde bucal. Escovação supervisionada, próteses... restabelecemos a função mastigatória. Para eles é uma possibilidade de inserção social. Dizem: 'quero colocar uma prótese'. Desejam isso mesmo, querem largar a rua, a droga, o álcool, buscam inserção social e trabalho. Procuram a odontologia nesse sentido." [P3]

"Eu faço higiene bucal deles, remoção de tártaro e polimento dos dentes. Oriento sobre saúde, higiene pessoal e oral. Faço palestra e acolhimento." [P10]

Depreendem-se nos depoimentos as ações educativas em saúde desenvolvidas nos eventos externos que ocorrem em espaços públicos, locais comuns de permanência da PSR:

"Realizamos uma ação/evento na Praça da Estação (referindo-se a um espaço público na cidade de Belo Horizonte). Falamos de DST (referindo-se às doenças sexualmente transmissiveis), aferimos a pressão arterial, abordamos os problemas de saúde $e$ a equipe da odontologia avalia e orienta." [P6]

Ações realizadas pela equipe de $\mathrm{SM}$, como apoio à eSF-PSR e eCR, foram enumeradas:

"Matriciamento dos casos junto às equipes e à rede socioassistencial, que envolve a equipe de abordagem de rua. Pensamos no atendimento clínico e no projeto terapêtico com a equipe que conduz o caso, para a melhor intervenção individual." [P1]

"Atuamos de forma intersetorial, ou seja, o serviço social, a clínica médica, a enfermagem, a zoonose e a educação, todos participam da elaboração do projeto terapêutico singular, a partir de cada pessoa. A saúde mental vem para escutar o que determinou a cisão dos laços com o outro e a escolha de viver na rua." [P12]
"Eu tenho trabalhado no matriciamento e no gerenciamento de casos junto a equipe de Consultório na Rua. Uma espécie de auditoria clínica para organizar um pouco e hierarquizar o cuidado, além de estabelecer prioridades." [P7]

Um dos profissionais da eCR reconhece seu papel ao descrever o processo de trabalho $\mathrm{e}$ as ações desenvolvidas:

"Nosso trabalho é itinerante, nós nos deparamos com o indivíduo em uso da droga, no local onde ele costuma ficar e perguntamos se podemos chegar, se ele aceita conversar. Trabalhamos com redução de danos. " [P8]

Outras ações elencadas foram as desenvolvidas por um dos integrantes da eCR, o arte educador, como estratégia de construção de vínculo e ressocialização da PSR:

"... atividades lúdicas, oficina de pintura, desenho e diversas outras atividades realizadas na rua." [P2]

"... tem o arte educador que é extremamente importante como integrante da equipe. Auxilia nas oficinas na rua, com crianças e adolescentes. Entendo como momento de fortalecimento de vínculo." [P12]

Ações relacionadas à remoção da referida população para a UBS ou para os serviços de urgência, realizadas pelos profissionais da eSF-PSR ou da eCR, quando em visita à PSR no território-rua, foram citadas:

"Encaminhar ao centro de saúde..., em caso de urgência e emergência encaminhamos para UPA, avaliamos dados vitais ... fazemos pequenos curativos." [P8]

As ações foram relacionadas pelos trabalhadores desde acolhimento, identificação, avaliação, até aquelas mais específicas e outras que permeiam qualquer uma delas, como a educação para a saúde. No entanto, mesmo cientes das atividades realizadas, foram destacados os 
dificultadores que tornam maiores os desafios a serem enfrentados.

\section{Enfrentando dificuldades e desafios no atendimento à PSR}

A maioria dos entrevistados revelou aspectos do perfil da PSR que impactam o atendimento, devido à dificuldade em localizar os indivíduos e à pressão para o atendimento imediato:

"Dificuldade de onde encontrar a pessoa $e$ de dar continuidade ao tratamento. As vezes inicia um tratamento e a pessoa muda de local." [P4]

"Outra dificuldade: eles são imediatistas. Sabem que não estarão aqui amanhã, naquele ponto, eles querem tudo no mesmo momento e estão sempre drogados ou alcoolizados. Por isso se marcar outro dia... virão as dificuldades de atendimento." [P3]

"A tuberculose tem uma incidência maior nessa população. É uma grande dificuldade o perfil, pois tem pouco comprometimento consigo mesmos. Então, conseguir medicar diariamente e observar, por seis meses, é quase um milagre." [P11]

Um dos profissionais ressalta que entender a complexidade dessa população e as especificidades de cada caso é crucial para o cuidado, mas exige criatividade e adaptação:

"Tudo da população de rua é específico, não existe padrão para se trabalhar. Lidamos com a criatividade pois cada um é de um jeito. Tem que discutir o caso e ver qual a maneira melhor de se trabalhar com aquela pessoa." [P4]

Dificuldades apontadas por alguns entrevistados relacionam-se às barreiras existentes no cuidado à PSR como a compreensão dos profissionais quanto à complexidade da SR, o discurso moral e a relação interprofissional, além da organização do serviço:

"Temos dificuldades com o trabalho intersetorial, a percepção de outros setores sobre os individuos nessa situação... eles tem seu momento próprio e não dão a mesma resposta que outra pessoa daria. As vezes a rede não entende. E para o projeto terapêutico singular é preciso afinação na equipe. Nem sempre o matriciamento ocorre como deveria, ou como desejamos." [P1]

"Às vezes é falta de perfil do profissional ou mesmo a insensibilidade de algumas áreas. Às vezes é falta de manutenção mais constante das equipes, já que a rotatividade de recursos humanos é alta. Além disso, o horário de atendimento de algumas áreas, eventualmente, não coincide com o do PSF. É preciso a conexão das duas equipes (referindo-se à eSF-PSR e eCR)." [P7]

"A maior dificuldade é o discurso moral que atravessa o cuidado... temos que ultrapassar isso para ver o indivíduo que precisa de cuidados, sem um préconceito." [P12].

"A forma que esse usuário é recebido no centro de saúde, também é uma dificuldade. Se ele achar que não foi bem recebido, não vai querer voltar." [P8].

As dificuldades elencadas envolveram desde a localização dos indivíduos em SR até a pressão para atendimento, que exige criatividade, adaptação e compreensão de especificidades. Obviamente, é preciso, na visão dos depoentes, libertar-se de preconceitos para vencer as barreiras para o cuidado em saúde.

\section{Discussão}

Por ocasião do estudo 1031 pessoas em SR estavam vinculadas à eSFPSR. Do total, 946 (92\%) homens adultos e 943 (91\%), com idade entre 20 a 59 anos. $\mathrm{Na}$ vivência dos profissionais de saúde que atendem tal população as questões mais comuns destacadas envolvem a saúde mental, uso e abuso de drogas lícitas e ilícitas e doenças infectocontagiosas. Dados governamentais corroboram os achados ao apontar que os principais 
problemas de adoecimento na PSR são transtorno mental, alcoolismo, tabagismo, TB e $\operatorname{AIDS}^{15}$.

Os resultados fizeram emergir as ações executadas no território/domicíliorua, por meio da "visita-rua", e tal espaço é apontado pela literatura como o local de permanência dos indivíduos, ponto de fixação e onde são encontrados ${ }^{10-11}$. Nestes locais foram destacadas as ações realizadas de prevenção de doenças e identificação de situações mais complexas com o devido encaminhamento, quando necessário. Percebe-se que os profissionais consideram o local de fixação dos indivíduos como ponto de partida para atendimento em saúde.

No atendimento à PSR identificar e cadastrar são tarefas essenciais que possibilitam informações e conhecimento do contexto social e tais atividades contam com o apoio de outras equipes ou instituições como o arte educador e a polícia militar, por exemplo. Emergem problemas de saúde, o acesso aos serviços e ao acolhimento institucional, além dos escassos recursos materiais e humanos que permitem um diagnóstico sobre essa população. Tais ações, preconizadas na eSF são executadas pelo ACS que vincula os usuários/famílias às equipes de $\mathrm{SF}^{11,15,22-23}$. Outras ações na rua são realizadas pela eSF-PSR, eCR e equipe de SM com destaque para a CIEC (Condução Involuntária para Exames e Cuidados), estratégia de abordagem e encaminhamento para um serviço de referência das pessoas com quadro clínico ou mental agudo sob risco de vida. São discutidas e programadas as ações e definidas as responsabilidades para cada profissional/serviço, de acordo com o caso em questão ${ }^{11}$, como pode ser evidenciado nos resultados.

$\mathrm{O}$ acolhimento foi enfatizado para o alcance da PSR e sua vinculação. Trata-se de tecnologia de relacionamento e de escuta qualificada, seja na UBS ou no território-rua para início do acompanhamento, compreensão da demanda dos indivíduos e sua inserção na RAS, com vistas à resolutividade assistencial. Acolher potencializa o processo terapêutico pela relação de afeto, confiança e vínculo e requer perfil profissional e disposição para tal habilidade $\mathrm{e}^{11,15,22-23}$. Os resultados ainda evidenciaram que o acolhimento, enquanto estratégia de vinculação e de consolidação do processo terapêutico, na visão dos profissionais, possibilita diálogo e reflexão sobre o estar na rua ou o desejo de sair da rua.

Em relação às patologias mais comuns emergiu a preocupação com a TB na PSR e sua identificação precoce. Os profissionais de saúde identificam a baixa autoestima dos indivíduos em SR e o pouco comprometimento consigo que pudesse sustentar a adesão ao tratamento. De fato, a incidência é maior nessa população, com chances de 48 a 67 mais vezes de adoecer comparando-se com a população em geral $^{11,15}$. Identificação, acompanhamento e tratamento até a cura da doença compõem as ações preconizadas pelo Ministério da Saúde, sendo a APS a porta de entrada e local privilegiado para a sua execução ${ }^{11,24}$. As metas propostas para o controle da TB, no Brasil, são de $85 \%$ de cura de casos novos da forma pulmonar, taxa de abandono abaixo de $5 \%$ e de detecção acima de $70 \%$, para que seja possível a redução da incidência em torno de $5 \%$ a $10 \%$ ao ano. Porém, para o grupo específico, são metas difíceis de serem alcançadas pela irregularidade e abandono do tratamento ${ }^{25-26}$. Entretanto, mesmo diante das dificuldades para se encontrar os indivíduos durante o tempo necessário para o uso de tuberculostáticos e para a própria adesão ao tratamento, as equipes, no cenário pesquisado, apostam no desejo e comprometimento da PSR em busca da saúde, o que tem possibilitado para alguns a alta por cura de $\mathrm{TB}$, reconhecido como 'quase um milagre'.

\section{Estudos} demonstram a potencialidade do trabalho em equipe, integrado e coordenado, com a ressalva de 
que é fundamental o apoio da gestão quanto aos recursos humanos e materiais, além da capacitação para atendimento ${ }^{23,26}$. Os resultados evidenciaram a importância de tal apoio, tanto por parte do gerente da UBS quanto da própria instituição na promoção da educação permanente, junto aos profissionais, que contemple temas relativos às especificidades da PSR, que desenvolva a criatividade necessária para que os serviços de saúde alcancem a população no seu local de fixação e que considerem o perfil social e epidemiológico específicos. Isso representaria a efetividade e eficiência esperada para a coisa pública no que tange ao atendimento dos indivíduos em SR.

Talvez por isso, a satisfação pessoal e gratificação dos profissionais no cuidado à PSR foram reveladas pela empatia e vínculos estabelecidos em função de uma relação humanizada e afetuosa que impacta positivamente nos resultados das ações de saúde. Para alguns autores seria o eixo do processo de cuidar voltado às especificidades do indivíduo. Isso se dá pela escuta, diálogo e apoio, além da disposição para acolher $\mathrm{e}$ atender as necessidades, com o direcionamento adequado e resolutivo na RAS. Uma experiência desafiante e recompensadora ${ }^{23,26}$. Ajudar, ouvir e acompanhar histórias de vida envolveram o cuidado prestado aos indivíduos em SR e foram percebidos como recompensa pelos depoentes que, de uma forma ou outra, têm seu trabalho reconhecido não apenas por si mesmos, mas pela população assistida.

Para os profissionais, contudo, o trabalho articulado em rede é fundamental considerando-se os DSS que impactam na saúde desta população e outras demandas, para além das questões de saúde, com destaque para questões econômicas e sociais. Alinhamento de estratégias e recursos de saúde, educação e assistência social tem sido considerados potencializadores do cuidado, diante da complexidade das demandas da $\mathrm{PSR}^{22-27}$. Torna-se possível intervir de forma resolutiva sobre os problemas de saúde e promover uma maior integração dessa população na rede de serviços ${ }^{12,22,27-28}$. De fato, os resultados apontaram falhas na articulação da rede intersetorial no atendimento à população com tantas especificidades. Seria necessário, além da muti, a interdisciplinaridade para que as competências se voltassem a um objetivo comum.

Defende-se, sobremaneira, considerar a importância de um provedor de cuidados primários, um serviço de saúde e profissionais na APS de referência, que sejam coordenadores do cuidado como prevê a Política Nacional da Atenção Básica, os atributos da APS e a própria ESF e, principalmente em se tratando da PSR, ao se considerar que tal nível de atenção à saúde deve ser a entrada do usuário na RAS. A experiência dos participantes, desse estudo, aponta neste sentido, devendo-se considerar que com a descentralização das ações voltadas a PSR, previstas nas políticas nacionais $\mathrm{e}$ municipais, pessoas em SR devem ser atendidas por profissionais das UBS do território-rua onde se fixaram. $\mathrm{O}$ encaminhamento dos indivíduos a outros pontos da rede deve partir de uma visão assistencial articulada e integral ${ }^{7,11,27,29}$. Os profissionais de saúde consideram que a PSR não é um grupo qualquer, que possa ser trabalhado 'em separado'. As ações devem estar pensadas, organizadas, planejadas e interligadas entre as equipes da APS quanto de outros pontos da RAS.

De fato, a inserção da eSB na ESF, por exemplo, para atendimento à PSR é fundamental, principalmente, junto aos usuários de drogas inalantes. Atividades realizadas na UBS ou nas praças, como orientações de higiene oral, distribuição de kits de cuidado bucal, profilaxia e tratamento clínico possibilitam o resgate da autoestima e autocuidado ${ }^{30}$. Estas foram atividades evidenciadas nos resultados para a RD, preocupação evidente dos profissionais ao considerar o ser humano em sua integralidade. 
Os entrevistados reconhecem a relação entre SB e saúde geral, sobretudo frente à alta vulnerabilidade social e às precárias condições higiênicas e utilizam cada oportunidade para educação sobre saúde em geral, além do cuidado com os dentes, remoção de tártaro e uso de próteses, quando indicado. De fato, a deficiência no autocuidado e no conhecimento sobre a SB refletem na não propriedade de escova dental e revelam mais uma vez a face cruel da exclusão social $^{31-32}$. Por outro lado, a reabilitação oral, que inclui o restabelecimento da função mastigatória, foi apontada e reconhecida pelos profissionais como demanda dos indivíduos para a reinserção social e abandono do uso de drogas. Neste sentido, algumas publicações indicam que a busca por melhorar a condição de SB relaciona-se à autopercepção sobre $\mathrm{O}$ próprio estado de saúde e o desejo de dentição funcional reconstruída para a 'retomada da vida' ${ }^{12-33}$. Não deixa de ser ocasião para o exame das questões socioeconômicas e psicossociais que se relacionam às intenções dessa população em se cuidar. Além de criar vínculos, contribuir com a cidadania e reinserção social da PSR e ampliar o acesso aos serviços de $\mathrm{SB}^{11,32}$.

As práticas educativas, como estratégia para promover saúde e prevenir doenças, foram citadas e devem favorecer o acesso, oportunizar a participação e o protagonismo da PSR, além de possibilitar a discussão de temas importantes ao seu contexto e necessidades ${ }^{30}$. As ações educativas descritas incluíram palestras, aferição de dados vitais e abordagem sobre diversos problemas de saúde que ocorreram tanto em ambiente interno, na UBS, quanto em praças públicas e outros locais, utilizados para permanência da PSR. É clara a percepção dos participantes de que educação em saúde e para a saúde devem sair da UBS e encontrar com os indivíduos em SR no local onde se fixam, normalmente. Para tanto, é necessário incorporar metodologias adaptadas à complexidade da vida social, capazes de promover discussão sobre os DSS, que favoreçam $\mathrm{o}$ protagonismo $\mathrm{e}$ empoderamento $^{34}$.

Os depoentes da equipe de SM revelaram ações que ultrapassam $\mathrm{O}$ atendimento clínico e suporte às eSF e eCR, que incluem apoio matricial, discussão de casos e elaboração de projeto terapêutico singular (PTS) realizados por profissionais de diversos setores (saúde, educação, vigilância e assistência social). Novamente, a multi e a interdisciplinaridade foram ressaltados.

O PTS refere-se a um conjunto de propostas de ações terapêuticas articuladas para um indivíduo, uma família ou um grupo, elaborado a partir da discussão coletiva entre profissionais de equipe interdisciplinar, em atividades de apoio matricial. Trata-se de uma ferramenta de cogestão e cuidado compartilhado, a partir da definição de objetivos comuns e do estabelecimento de tarefas correlacionadas e pactuadas em equipe. Ele se desenvolve em quatro momentos, a saber: diagnóstico, definição de metas, divisão de responsabilidade e reavaliação, geralmente proposto para os casos mais complexos ${ }^{35-}$ 37. O apoio matricial, também chamado de matriciamento, acena para uma 'tecnologia de arranjo organizacional' que possibilita o suporte técnico de áreas especializadas junto às equipes da APS. Caracteriza-se pela relação multi-interdisciplinar entre equipes de SF e de apoio e se configura nas dimensões técnico-pedagógica e assistencial. A primeira inclui ações conjuntas entre profissionais das equipes e os de apoio que podem ocorrer durante a discussão de temas e/ou a partir do atendimento individual, domiciliar, atividade coletiva e ações no território, de forma compartilhada. A dimensão assistencial refere-se às intervenções diretas das equipes de apoio junto aos usuários, de acordo com as especificidades da categoria, que podem ocorrer de forma individual ou coletiva. Além disso, o matriciamento pode ser uma importante 
estratégia de educação permanente, por considerar a oportunidade de compartilhar saberes e práticas que estimulam o aprender-fazer em conjunto ${ }^{35-37}$.

Desta forma, o matriciamento acontece a partir da assistência conjunta e compartilhada entre um apoiador/especialista e uma equipe de referência, como ferramenta de transformação que amplia a integralidade e a cogestão do cuidado, da universalidade do acesso e de tratamento individualizado, além de aumentar o escopo de ações e a capacidade resolutiva da APS $^{35-37}$. Expressões como 'intervenção individual' e 'a partir de cada pessoa' puderam ser observadas nos depoimentos e denotam a visão dos depoentes sobre o cuidado singular.

Assim sendo, o apoio matricial favorece a superação da fragmentação do conhecimento, a ampliação da capacidade de análise e a ação dos profisssionais no sentido da coprodução de saúde e da autonomia das equipes matriciadas e dos indivíduos-alvo do cuidado ${ }^{35-37}$. Especificamente, a participação da equipe de SM fez emergir subjetividades do contexto de vida da PSR, a partir da escuta, com auxílio da equipe de abordagem de rua e compreensão sobre o que teria determinado ou contribuído para a cisão dos laços, as motivações e a escolha de vida na rua.

As eCR, por sua vez, são a ponte entre a PSR e as equipes da APS, frente às ações para $\mathrm{RD}$ e que fortalecem a autonomia em diversos cenários na rua, principalmente em relação ao uso de drogas ${ }^{11,16,17}$. Reduzir danos, papel reconhecido pelos participantes, representa uma estratégia para pessoas que desejam e não conseguem diminuir/interromper o uso de drogas, no sentido de minimizar os prejuízos gerados pelo uso abusivo. São trabalhados aspectos inerentes ao uso de drogas e sua influência na saúde, a retomada do autocuidado e da vida social, com transferência de foco da droga para o foco da vida. O cenário de atuação das
eCR tanto pode ser onde a PSR permanece a maior parte do tempo na rua, considerado como local de fixação, quanto $o$ de circulação dessa população, sendo que diversas ações, muitas vezes, precisam ser articuladas com outros pontos da RAS e/ou com instituições da educação, do trabalho e da promoção social ${ }^{10-11,16-17,28,38-39}$. Tal cenário de fixação da PSR foi reconhecido pelos profissionais como melhor local para a atuação itinerante das eSF-PSR e eCR, que possibilita abordar aqueles que desejam, pelo menos, conversar. A partir daí, vínculos podem se formar nesta forma de acolhimento.

No entanto, existem fragilidades ao se buscar a RD, pois implica comprometimento integrado e preparação dos trabalhadores de saúde, envolvimento do indivíduo e financiamento para manutenção das equipes ${ }^{30,38,40}$. Depreendem-se nos resultados as atividades realizadas, como jogos, pintura e desenhos que demandam certa logística e capacitação dos instrutores para o alcance dos objetivos. Pesquisas demonstram que pintura, jogos, dança e música são recursos que possibilitam trabalhar conteúdos emocionais não explorados. Somam-se os incentivos às novas percepções em relação ao meio e a si mesmo, a construção coletiva de sentido que potencializa a individualidade, estimula a autonomia e empoderamento, além de contribuir para inclusão social ${ }^{41-42}$. Embora as atividades citadas possam ser desenvolvidas pela equipe em geral, no cenário do presente estudo, são realizadas pelo arte educador.

Os resultados encontrados e corroborados pela literatura, em relação ao serviços de saúde mais procurados pela PSR, ressaltam aqueles relacionados a atendimentos de urgência e emergência. Mas, há dificuldades no atendimento a essa população devido à deficiência de capacitação e do preconceito. Infraestrutura deficitária e inadequação de locais para o restabelecimento pós-alta hospitalar também são evidenciados ${ }^{7,11,43}$. Para enfrentar a situação urge investir em 
ações multiprofissionais e intersetoriais de cuidados primários. A literatura demonstra ainda impactos positivos na saúde da PSR, com redução de uso de serviços de urgência, frente à facilidade de acesso aos serviços, estabelecimento de vínculo e engajamento às ações de cuidado e autocuidado $27,29,43$. Ao mesmo tempo que reconhecem eventuais preconceitos, os entrevistados enfatizaram a necessidade de quebra de certos paradigmas para universalizar o cuidado em saúde, em seu verdadeiro sentido.

Dentre outros dificultadores os resultados destacaram aqueles relacionados ao acompanhamento da PSR em função do seu perfil específico e o desejo de atendimento imediato. Os profissionais, por sua vez, percebem que diante da demora no atendimento às demandas perde-se a oportunidade para a promoção da saúde e a prevenção de doenças. Os mesmos problemas são citados por outros pesquisadores como ausência de endereço fixo, alta mobilidade e deslocamento e ausência ou baixa adesão a tratamentos, que se constituem desafios para o cuidado $^{11,22,44}$. Entende-se não haver uma única forma de abordagem e acompanhamento, sendo fundamental compreender suas especificidades, discutir em equipe e compartilhar possibilidades. Cabe ressaltar que o processo de cuidar não deve ser engessado, padronizado e adaptações são necessárias, além de criatividade, como evidenciado nos resultados.

Por outro lado, diferentes percepções dos participantes em relação à PSR contribuem para a dificuldade de engajamento e responsabilização pelo cuidado a essa população. Aponta-se a existência de muitas barreiras pessoais e estruturais que dificultam ou impedem o acesso de grupos marginalizados aos cuidados primários em saúde ${ }^{22,44}$. Perfil inadequado para trabalhar com PSR, a alta rotatividade e o discurso moral que norteiam a prática dos profissionais de saúde em diversos serviços dificultam o matriciamento e a construção efetiva dos PTS. Outras pesquisas ainda acrescentam a dificuldade na construção de vínculos, a precariedade na organização do serviço, a exigência de documentação e o não reconhecimento da PSR como indivíduos com direitos como qualquer pessoa $22,28,44$. Os participantes reconhecem as ações realizadas, mas, ao mesmo tempo, identificam as dificuldades pessoais, na equipe e gerenciais para a consecução dos objetivos propostos e maior efetividade nas respostas aos indivíduos atendidos.

Enfim, há que se moldar um perfil para aquele que atende indivíduos em SR que inclua habilidade para a formação de vínculos, visão compreensiva, orientação ética, disposição para escuta, sensibilidade e destreza para a comunicação. Competências capazes de identificar as reais necessidades da pessoa na perspectiva do cuidado integral ${ }^{22-23,26}$, destacadas nos resultados encontrados.

Isso posto, entende-se a importância devida e requerida em relação a conhecer cada local de atuação e a população atendida para, de posse de tal diagnóstico, estabelecer cuidado que faça diferença no contexto individual e na saúde da população como um todo. Quanto mais claro e amplo o diagnóstico e quanto mais permeável a RAS, mais profundo o alcance das ações e mais facilmente serão superadas as dificuldades.

\section{Conclusão}

Conhecer as ações desenvolvidas em qualquer lugar do mundo onde existam pessoas em SR tem como implicação para a prática de cuidados em saúde o olhar que considere as diferenças, respeite a autonomia e as demandas individuais. As ações desenvolvidas foram reconhecidas pelos profissionais, mesmo diante de dificuldades que existem e sempre existirão, mas a adequação dos processos de trabalho passa a ser reclamada em 
relação à capacitação dos profissionais e otimização das ações executadas.

O planejamento do que precisa ser realizado com vistas à PS e prevenção da doença deve considerar a assistência integrada, multi e interdisciplinar e, o mais relevante, sempre em rede. Ressalta-se que a abordagem no contexto de vida é experiência que facilita o vínculo.

Embora os resultados desse estudo apontem para aspectos positivos, exitosos no cuidado a PSR, construída ao longo de anos de experiência junto a essa população, observam-se ainda problemas a serem enfrentados, descritos de maneira clara e que reclamam sua gestão, preferencialmente, compartilhada.
O estudo possui limitações por ter sido realizado em apenas uma UBS, apesar de se constituir em unidade referência para a PSR, porém, a pesquisa qualitativa não busca a generalização de resultados. $\mathrm{O}$ que se buscou foi a vivência dos profissionais de saúde no atendimento aos indivíduos em SR. Entretanto, novos estudos que abordem questões mais específicas relacionadas à SR, como a percepção individual dos envolvidos, suas relações familiares para o cuidado em saúde ou, ainda, os eixos centrais de capacitação em rede poderiam contribuir para outras estratégias de atendimento, captação, inserção e vinculação destes indivíduos nos serviços de saúde ao redor do mundo.

\section{Referências}

1. Organização das Nações Unidas. Conference of European Statisticians. Twelfth Meeting. Geneva, 2009. Enumeration of homeless people. Counting homeless people in the 2010 census round: use of enumeration and register-based methods. [Internet]. Disponível em: https://unstats.un.org/unsd/censuskb20/KnowledgebaseArticle10634.aspx

2. Culhane D. Leave No One Behind: Disaggregating Homelessness In Support of SDG 11. [Internet]. Expert Group Meeting On Data Disaggregation. 2016, New York.

ESA/STAT/AC.320/24. Disponível em: https://unstats.un.org/sdgs/files/meetings/egmdata-dissaggregation/PPT24-Culhane.pdf .

3. Organização das Nações Unidas. Report of the Special Rapporteur on adequate housing as a component of the right to an adequate standard of living, and on the right to nondiscrimination in this context. 2015. [Internet]. Disponível em: https://www.ohchr.org/en/issues/housing/pages/housingindex.aspx

4. Neves-Silva $\mathrm{P}$, Heller L. O direito humano à água e ao esgotamento sanitário como instrumento para a promoção da saúde de populações vulneráveis. Ciência \& Saúde Coletiva. 2016;21(6):1861-70.

5. Garey L, Reitzel LR, Kendzor DE, Businelle MS. The Potential Explanatory Role of Perceived Stress in Associations Between Subjective Social Status and Health-Related Quality of Life Among Homeless Smokers. Behavior Modification. 2016:40(1-2):303-24.

6. Romaszko J, Kuchta R, Opalach C, et al. Socioeconomic Characteristics, Health Risk Factors and Alcohol Consumption among the Homeless in North-Eastern Part of Poland. Central European Journal of Public Health. 2017;25(1):29-34.

7. Smith OM, Chant C, Burns KEA, et al. Characteristics, clinical course, and outcomes of homeless and non-homeless patients admitted to ICU: A retrospective cohort study. PLoS One. 2017;12(6):e0179207.

8. Brasil. Ministério de Desenvolvimento Social e Combate à Fome. Decreto No 7.053, de 23 de dezembro de 2009. Institui a Política Nacional Para Inclusão Social da População em Situação de Rua e seu Comitê Intersetorial de Acompanhamento e Monitoramento, e dá outras providências. [Internet]. Diário Oficial da União, Brasília, DF, 23 dez. 2009. Disponível em: http://www.planalto.gov.br/ccivil_03/_ato2007$\underline{\text { 2010/2009/decreto/d7053.htm }}$ 
9. Natalino M. Estimativa da população em situação de rua no Brasil (setembro de 2012 a março de 2020). Ipea - Instituto de Pesquisa Econômica Aplicada. Diretoria de Estudos e Políticas Sociais (DISOC). Nota Técnica No 73, jun. 2020. [Internet]. Livraria Ipea. Disponível em: https://www.ipea.gov.br/portal/index.php?option=com content\&view=article\&id $=35812$

10. Paiva IKS, Lira CDG, Justino JMR, Miranda MGO, Saraiva, AKM. Direito à saúde da população em situação de rua: reflexões sobre a problemática. Ciência \& Saúde Coletiva. 2016;21(8):2595-606.

11. Brasil. Ministério da Saúde. Secretaria de Atenção a Saúde. Departamento da atenção Básica. Manual sobre o cuidado à saúde junto à população em situação de rua. [Internet]. Brasília, DF. 2012. Disponível em: http://189.28.128.100/dab/docs/publicacoes/geral/manual_cuidado populalcao_rua.pdf

12. Borysow IC, Conill EM, Furtado JP. Atenção à saúde de pessoas em situação de rua: estudo comparado de unidades móveis em Portugal, Estados Unidos e Brasil. Ciência \& Saúde Coletiva. 2017;22(3):879-90.

13. Minas Gerais. Tribunal de Justiça de Minas Gerais. Assessoria de Comunicação Institucional - Ascom. Minas tem mais de 18 mil pessoas em situação de rua [Internet] Novo Portal TJMG. 15 jul. 2020. Disponível em: https://www.tjmg.jus.br/portaltjmg/noticias/minas-tem-mais-de-18-mil-pessoas-em-situacao-de-rua.htm\#.YDVkaNhKjIV

14. Belo Horizonte. Prefeitura de Belo Horizonte. Terceiro censo da População em Situação de Rua e Migrantes. [Internet]. 2014. Disponível em:

http://portalpbh.pbh.gov.br/pbh/ecp/noticia.do?evento $=$ portlet\&pAc $=$ not\&idConteudo $=154$ $144 \& p I d P 1 c=\& a p p=$ salanoticias

15. Brasil. Ministério da Saúde. Secretaria de Gestão Estratégica e Participativa. Departamento de Apoio à Gestão Participativa. Saúde da população em situação de rua: um direito humano. [Internet]. Brasília, DF, 2014. Disponível em: http://bvsms.saude.gov.br/bvs/publicacoes/saude_populacao_situacao_rua.pdf

16. Abreu D, Oliveira WF. Atenção à saúde da população em situação de rua: um desafio para o Consultório na Rua e para o Sistema Único de Saúde. Cadernos de Saúde Pública. 2017;33(2):e00196916.

17. Brasil. Ministério da Saúde. Portaria No 122, de 25 de Janeiro de 2011. Define as diretrizes de organização e funcionamento das Equipes de Consultório na Rua. [Internet]. Diário Oficial da União, Brasília, DF, 25 jan. 2012. Disponível em: http://bvsms.saude.gov.br/bvs/saudelegis/gm/2012/prt0122_25_01_2012.html

18. Brasil. Ministério da Saúde. Secretaria de Atenção à Saúde. Departamento de Atenção Básica. Departamento de Ações Programáticas Estratégicas. Coordenação de Área Técnica de Saúde Mental. Nota Técnica Conjunta/2012. Adequação dos Consultórios de Rua e Implantação de novas equipes de Consultório na Rua frente às diretrizes de funcionamento das equipes de Consultório na Rua, estabelecidas pelas portarias 122 e 123, de 25 de janeiro de 2012. [Internet]. Brasília, DF. 2012. Disponível em: http://189.28.128.100/dab/docs/geral/20120412004951716.pdf

19. Minayo MCS. O desafio do conhecimento. Pesquisa qualitativa em saúde. $13^{a}$ ed. São Paulo: Hucitec - Um livro, 2014. 408p.

20. Moreira H, Caleffe LG. Metodologia da pesquisa para o professor pesquisador. $2^{\text {a }}$ ed. Rio de Janeiro: Lamparina, 2008. 248p.

21. Bardin L. Análise de conteúdo. Lisboa: Edições 70, 2011. 280p.

22. Costa KMR, Sousa JJS, Rocha RB, Lino MRB, Brito GV, Sousa NF. Cuidado à saúde da pessoa em situação de rua: debate e relatos para uma abordagem intersetorial. Saúde e Pesquisa. 2017;10(2):251-58. 
23. van Wijk LB; Mângia EF. O cuidado a pessoas em situação de rua pela Rede de Atenção Psicossoacial da Sé. Saúde em Debate. 2017;41(115):1130-42.

24. Brasil. Ministério da Saúde. Secretaria de Vigilância em Saúde. Departamento de Vigilância Epidemiológica. Coordenação Geral do Programa Nacional de Controle de Tuberculose. Tratamento Diretamente Observado (TDO) da Tuberculose na Atenção Básica: Protocolo de Enfermagem. [Internet]. Brasília, DF. 2011. Disponível em: http://bvsms.saude.gov.br/bvs/publicacoes/tratamento diretamente observado tuberculose. pdf

25. Brasil. Ministério da Saúde. Secretaria de Vigilância em Saúde. Boletim Epidemiológico: Detectar, tratar e curar: desafios e estratégias brasileiras frente à tuberculose. [Internet]. Brasília, DF. 2015. Disponível em: http://portalarquivos.saude.gov.br/images/pdf $/ 2015 / \mathrm{marco} / 25 /$ Boletim-tuberculose2015.pdf

26. Alecrim TFA, Mitano F, Reis, AA, Roos CM, Palha PF, Protti-Zanatta ST. Experiência dos profissionais de saúde no cuidado da pessoa com tuberculose em situação de rua. Revista da Escola de Enfermagem da USP. 2016;50(5):808-15.

27. Jego M, Abcaya J, Stefan DE, Calvet-Montredon C, Gentile S. Improving Health Care Management in Primary Care for Homeless People: A Literature Review. International Journal of Environmental Research and Public Heakth. 2018;15(2):309.

28. Kami MTM, Larocca LM, Chaves MMN, Piosiadlo LCM, Albuquerque GS. Saberes ideológicos e instrumentais no processo de trabalho no Consultório na Rua. Revista da Escola de Enfermagem da USP. 2016;50(3):440-7.

29. Roche MA, Duffield C, Smith J, et al. Nurse-led primary health care for homeless men: a multimethods descriptive study. International Nursing Review. 2018;65(3):392-99.

30. Engstrom EM, Teixeira MB. Equipe "Consultório na Rua" de Manguinhos, Rio de Janeiro, Brazil: práticas de cuidado e promoção da saúde em um território vulnerável. Ciência \& Saúde Coletiva. 2016;21(6):1839-48.

31. Jones K, Brennan D, Parker E, et al. Are oral health-related self-efficacy, knowledge and fatalism indicators for non-toothbrush ownership in a homeless population? Community Dental Health. 2016;33(2):48-53.

32. Coles E, Freeman R. Exploring the oral health experiences of homeless people: a deconstruction-reconstruction formulation. Community Dentistry Oral Epidemiology. 2016;44(1):53-63.

33. Santos LM, Noro LRA, Roncalli AG, Teixeira AKM. Autopercepção sobre saúde bucal e sua relação com utilização de serviços e prevalência de dor de dente. Revista Ciência Plural. 2016;2(2):14-27.

34. March S, Martín MJ, Gomis IM, et al. ¿Qué hacemos en el barrio? Descripción de las actividades comunitarias de promoción de la salud en atención primaria: proyecto frAC. Gaceta Sanitaria. 2014;28(4):267-73.

35. Hirdes A. A perspectiva dos profissionais da Atenção primária à Saúde sobre o apoio matricial em saúde mental. Ciência \& Saúde Coletiva. 2015;20(2):371-82.

36. Lima M, Dimenstein M. O apoio matricial em saúde mental: uma ferramenta apoiadora de atenção à crise. Interface (Botucatu). 2016;20(58):625-35.

37. Klein AP, d'Oliveira AFPL. O "cabo de força" da assistência: concepção e prática de psicólogos sobre o apoio matricial no Núcleo de Apoio à Saúde da Família. Cadernos de Saúde Pública. 2017;33(1):e00158815.

38. Abreu D. Consultório na Rua e Redução de Danos: estratégias de ampliação da vida. Revista Brasileira de Medicina de Família e Comunidade. 2017;12(39):1-2.

39. Schervinski AC, Merry CN, Evangelista IC, Pacheco VC. Atenção à saúde da população em situação de rua. Revista Eletrônica de Extensão - Extensio. 2017;14(26):55-64. 
40. Silva FP, Frazão IS, Linhares FMP. Práticas de saúde das equipes dos Consultórios de Rua. Cadernos de Saúde Pública. 2014;30(4):805-14.

41. Ibiapina ARS, Monteiro, CFS, Alencar DC, Fernandes MA, Costa Filho AAI. Oficinas terapêuticas e as mudanças sociais em pacientes com transtornos mentais. Escola Anna Nery. 2017;21(3):e20160375.

42. Farre AGMC, Pinheiro PNC, Vieira NFC, Gubert FA, Alves MDS, Monteiro EMLM. Promoção da saúde do adolescente baseada na arte/educação e centrada na comunidade. Revista Brasileira de Enfermagem. 2018;71(1):26-33.

43. Gabrielian S, Chen JC, Minhaj BP, et al. Feasibility and Acceptability of a Colocated Homeless-Tailored Primary Care Clinic and Emergency Department. Journal of Primary Care \& Community Health. 2017;8(4):338-44.

44. O'Donnell P, Tierney E, O'Carroll A, Nurse D, MacFarlane A. Exploring levers and barriers to accessing primary care for marginalised groups and identifying their priorities for primary care provision: a participatory learning and action research study. International Journal for Equity in Health. 2016;15(1):197.

\section{Como citar este artigo:}

Laura C, Cruz AD, Salles MM, Perillo RD, Torres LM, Almeida SP. Cuidados primários em saúde na atenção à população em situação de rua. Rev. Aten. Saúde. 2021; 19(67): 234-250. 